\title{
Were planetesimals formed by dust accretion in the solar nebula?
}

\author{
Minoru Sekiya ${ }^{1}$, and Hidenori Takeda ${ }^{2}$ \\ ${ }^{1}$ Department of Earth and Planetary Sciences, Faculty of Sciences, 33 Kyushu University \\ Hakozaki, Fukuoka 812-8581, Japan \\ ${ }^{2}$ Department of Aeronautics and Astronautics, Graduate School of Engineering, Kyoto University, \\ Sakyo-ku, Kyoto 606-8501, Japan
}

(Received March 20, 2003; Revised June 3, 2003; Accepted June 5, 2003)

\begin{abstract}
The growth of meter-sized bodies in the solar nebula by dust accretion is examined. The meter-sized bodies have velocity about $50 \mathrm{~m} / \mathrm{s}$ relative to the gas and small dust aggregates. When a small dust aggregate hits a meter-sized body, the aggregate breaks into dust monomers. These monomers accrete onto the body after several bouncing as proposed by Wurm et al., Icarus (2001), if the mean free path of the gas molecules is larger than the radius of the body. On the other hand, the monomers never hit the surface of the body again, if the body is much larger than the mean free path of the molecules. The sizes of bodies would be limited to the order of 10 times the mean free path. Kilometer-sized planetesimals were hardly formed by dust accretion in the region within 5 AU from the sun where the mean free path is less than $1 \mathrm{~m}$. The planetesimals were probably formed by the gravitational instabilities in this region.
\end{abstract}

Key words: Planets, solar system, solar nebula, planetesimals.

\section{Introduction}

Terrestrial planets are considered to have been formed through continuous collisional coagulations of planetesimals due to mutual gravitational force (Safronov, 1969; Hayashi et al., 1985). The collisional coagulation due to mutual gravity occurs for planetesimals whose radii are $\gtrsim 1 \mathrm{~km}$ and the escape velocities are $\gtrsim 1 \mathrm{~m} / \mathrm{s}$. The initial radii of solid dust particles (called as monomers in the following) in the protoplanetary disk are probably on the order of $1 \mu \mathrm{m}$. Thus, there should have been processes other than the gravitational coagulation for solid bodies to grow their sizes from $\sim 1 \mu \mathrm{m}$ to $\sim 1 \mathrm{~km}$.

Formerly, fragmentation of a thin dust layer around the midplane of the protoplanetary disk due to self-gravity was considered to be the most promising process of formation of a lot of km-sized planetesimals (Safronov, 1969; Goldreich and Ward, 1973; Coradini et al., 1981; Sekiya, 1983). However, shear-induced turbulence in the dust layer may prevent the dust aggregates from settling sufficiently to reach the critical density of the gravitational fragmentation (Weidenschilling, 1980, 1984; Cuzzi et al., 1993; Weidenschilling and Cuzzi, 1993; Sekiya, 1998).

Recently, an interesting process of growth of dust aggregates in the nebular gas was proposed by Wurm et al. (2001a, b). When a small dust aggregate hits a large dust aggregate with a velocity $\gtrsim 1 \mathrm{~m} / \mathrm{s}$, the former would be disrupted into monomers; they are subsequently dragged by the gas flow toward the large aggregate, and after bouncing several times on the surface of the large aggregate, they eventually accrete onto the surface. Wurm et al. $(2001 \mathrm{a}, \mathrm{b})$ have

Copy right(C) The Society of Geomagnetism and Earth, Planetary and Space Sciences (SGEPSS); The Seismological Society of Japan; The Volcanological Society of Japan The Geodetic Society of Japan; The Japanese Society for Planetary Sciences. shown from their experiments that this process is very efficient so that a small dust aggregate can accrete onto a larger body even if the collisional velocity is $\gtrsim 1 \mathrm{~m} / \mathrm{s}$. The target which was used to simulate a large aggregate in their experiments had a size comparable to the mean free path of gas molecules; that is, their experiments were done in the transition regime between the continuous fluid and the free molecule flow. Thus, bodies in the solar nebula are expected to grow until their sizes become on the order of the mean free path through the above mentioned process. However, it is not known whether the bodies can grow further to sizes much larger than the mean free path through the accretion of the dust aggregates and monomers.

In this paper, we consider the growth of a spherical body much larger than the mean free path, but much smaller than $1 \mathrm{~km}$; thus, the coagulation due to gravity does not occur. Then the flow around the body is obtained by hydrodynamic equations. In a flow around a body much larger than the mean free path, gas molecules do not move ballistically. They rather flow hydrodynamically around the body. After a dust aggregate collides and breaks up into monomers, they may be dragged away from the sphere by the gas flow. Thus it is expected that the gas drag prevents the accretion of small aggregates onto larger bodies, as already pointed out by Wurm et al. (2001a) in their last paragraph. In order to elucidate this process, we solved trajectories of small dust aggregates and monomers in hydrodynamic flow around a spherical body much larger than the mean free path.

Since the mean free path of gas molecules $l_{g} \gtrsim 1 \mathrm{~m}$ in the outer solar nebula (i.e. the heliocentric distance $r \gtrsim 5 \mathrm{AU}$ ), the hydrodynamic approach is not relevant to bodies with $\lesssim 100 \mathrm{~m}$ for which the collisional coagulation due to mutual gravity can be neglected. Thus, we restrict ourselves to treat the inner solar nebula $(r \lesssim 5 \mathrm{AU})$ in this paper. 


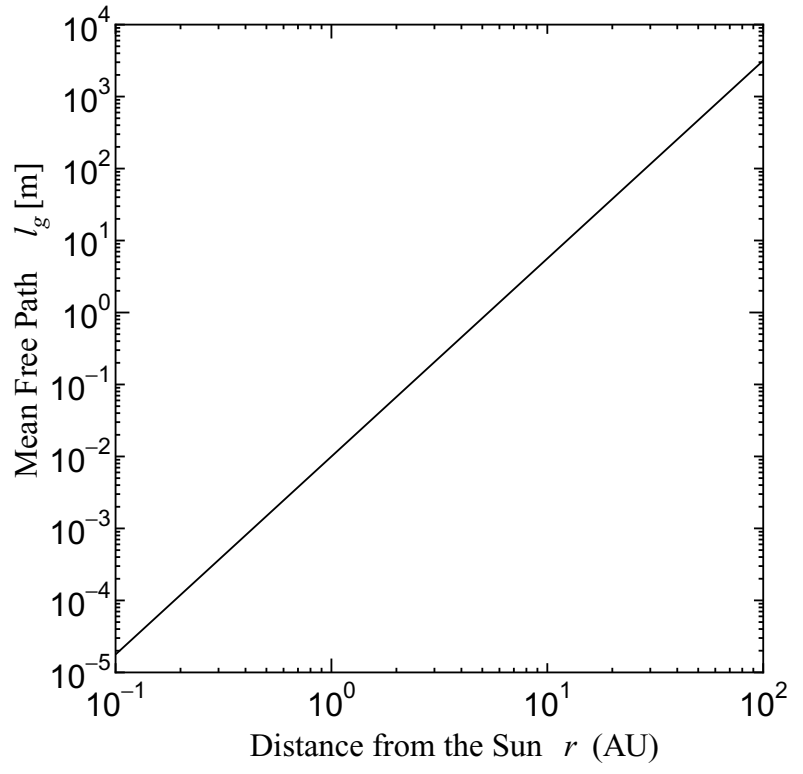

Fig. 1. The mean free path of gas molecules in the minimum mass solar nebula.

In Section 2, the model used in this paper is described, and the velocity of a meter-sized body and the Reynolds number of the flow around the body are obtained. In Section 3, the trajectories of small dust aggregates and monomers around a large target body is calculated. In Section 4, implications on planet formation from our results are discussed. In Section 5 , conclusions are summarized.

\section{The Velocity of a Meter-Sized Spherical Body in the Nebular Gas and the Reynolds Number of the Flow around the Body}

We use the minimum mass model (Hayashi, 1981; Hayashi et al., 1985) as a representative model of the solar nebula. The gas density around the midplane is given by

$$
\rho_{g}=1.4 \times 10^{-6}(r / \mathrm{AU})^{-11 / 4} \mathrm{~kg} / \mathrm{m}^{3} .
$$

The mean free path of the gas molecules around the midplane of the nebula is given by (see Fig. 1)

$l_{g}=\left[2^{1 / 2} \sigma_{m o l}\left(\rho_{g} / \mu_{m o l} m_{H}\right)\right]^{-1}=1.0 \times 10^{-2}(r / \mathrm{AU})^{11 / 4} \mathrm{~m}$,

where $m_{H}$ is the mass of a hydrogen atom, and $\sigma_{m o l}$ and $\mu_{m o l}$ are the mean cross section and the mean molecular weight of molecules. We take $\sigma_{m o l}=2 \times 10^{-19} \mathrm{~m}^{2}$ and $\mu_{m o l}=2.34$, respectively.

In this paper, we restrict ourselves to the case where the dust density, i.e. the total mass of dust aggregates and monomers in unit volume of the nebula, is much smaller than the gas density. Then the revolution velocity of the nebular gas $v_{g}$ is slightly slower than the circular Kepler velocity $v_{K}$ due to outward pressure gradient; $v_{g}=(1-\eta) v_{K}$, where,

$$
\eta=-\frac{1}{2 r \Omega_{K}^{2} \rho_{g}} \frac{\partial P_{g}}{\partial r},
$$

where $P_{g}$ is the gas pressure and $\Omega_{K}$ is the circular Keplerian angular velocity (Adachi et al., 1976; Weidenschilling, 1977; Nakagawa et al., 1986).

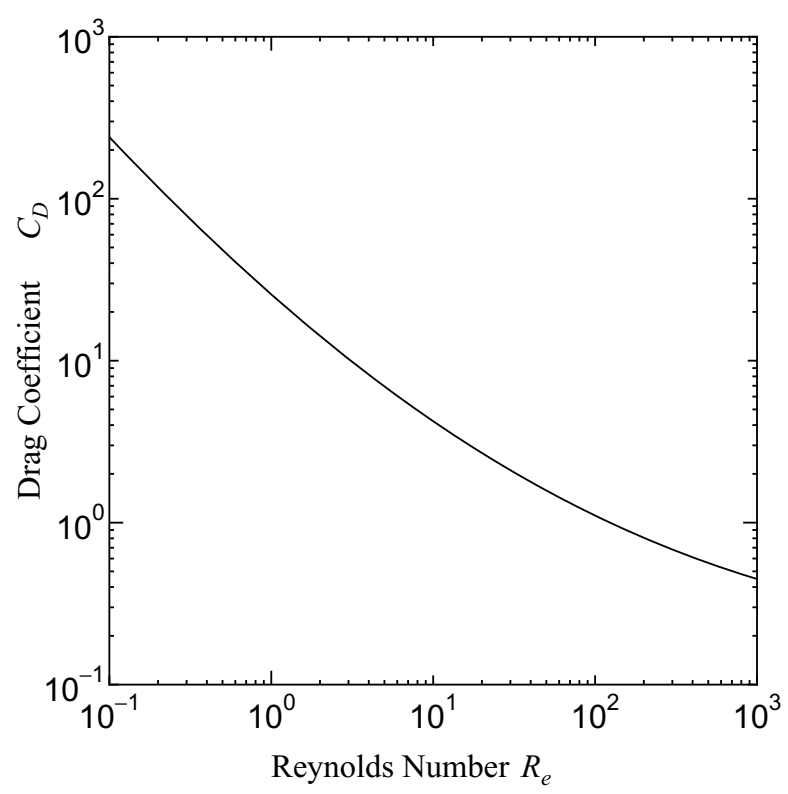

Fig. 2. The gas drag coefficient as a function of the Reynolds number.

We consider whether the growth of a spherical body (hereafter called SB) much larger than the mean free path by accreting small dust aggregates and monomers is possible. The gas flow around a SB is a classical issue and a number of experiments (Taneda, 1956; Magarvey and Bishop, 1961; Goldburg and Florsheim, 1966; Nakamura, 1976) as well as numerical simulations (Rimon and Cheng, 1969; Kalro and Tezduyar, 1998; Lee, 2000) have been done. The flow is characterized by the Reynolds number $R_{e}=2 R v_{\infty} / v$, where $R$ is the radius of the $\mathrm{SB}, v_{\infty}$ is the upstream velocity, and $v$ is the kinematic viscosity. The flow is steady and axisymmetric as long as $R_{e}<130$. We restrict ourselves to this regime in this paper. The drag force on SB is usually written in the form

$$
F_{D}=\frac{1}{2} C_{D} \rho_{g} v_{\infty}^{2} \pi R^{2}
$$

where $C_{D}$ is a non-dimensional coefficient (the drag coefficient). As shown in Fig. 2, $C_{D}$ is a function of $R_{e}$, which is obtained by experiments and/or numerical simulations (e.g., Rimon and Cheng, 1969). The friction time of SB is given by

$$
t_{f S B}=(4 \pi / 3) R^{3} \rho_{S B} v_{\infty} / F_{D},
$$

where $\rho_{S B}$ is the density of SB.

The motion of a body in the solar nebula is determined by the gravitational force of the sun as well as other bodies, the gas drag force, and the effect of collision with other bodies. We here neglect the random velocity excited by the gravitational force of other bodies, because we consider the stage before $\mathrm{km}$-sized planetesimals are formed and treat bodies with radii $\lesssim 10 \mathrm{~m}$. We also neglect the effect of collisions, since the collisions between large bodies under consideration are not so frequent. In that situation, Adachi et al. (1976) and Weidenschilling (1977) investigated the motion of solid particles in the solar nebula. The radial and azimuthal components of the velocity of SB relative to the nebular gas, $v_{r}$ and $v_{\phi}-v_{g}$, respectively, are given by 


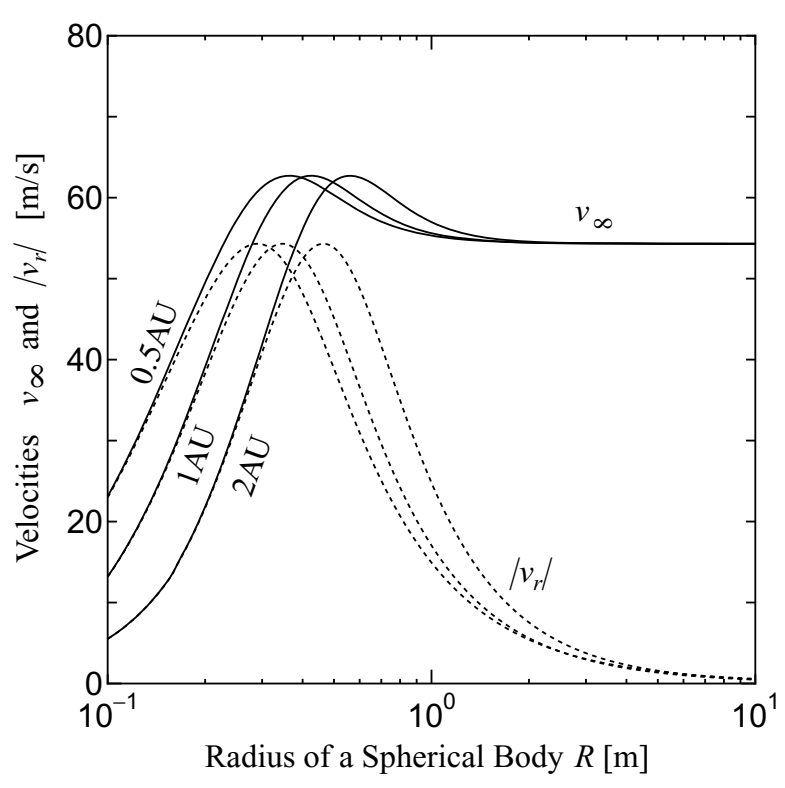

Fig. 3. The velocity of a solid sphere with radius $R$ for $r=0.5,1$, and 2 AU. The solid curves show the absolute value of the velocity relative to the gas, $v_{\infty}$, and the dashed curves show the radial infall velocity toward the sun, $\left|v_{r}\right|$.

Eqs. (5.5) to (5.7) and (5.9) of Adachi et al. (1976):

$$
v_{r}=-\frac{2\left(\Omega_{K} t_{f S B}\right)^{-1}}{1+\left(\Omega_{K} t_{f S B}\right)^{-2}} \eta v_{K},
$$

and

$$
v_{\phi}-v_{g}=\frac{1}{1+\left(\Omega_{K} t_{f S B}\right)^{-2}} \eta v_{K} .
$$

The magnitude of the relative velocity is

$$
\begin{aligned}
v_{\infty} & =\left[v_{r}^{2}+\left(v_{\phi}-v_{g}\right)^{2}\right]^{1 / 2}, \\
& =\frac{\left[1+4\left(\Omega_{K} t_{f S B}\right)^{-2}\right]^{1 / 2}}{1+\left(\Omega_{K} t_{f S B}\right)^{-2}} \eta v_{K} .
\end{aligned}
$$

Equations (4), (5) and (8) are coupled each other (note that $C_{D}$ is also a function of $v_{\infty}$ through $R_{e}$ ). The values of $v_{r}$ and $v_{\infty}$ are drawn in Fig. 3 and $R_{e}$ in Fig. 4 as functions of $R$ for $r=0.5,1$ and 2 AU. Note that values in these figures are calculated with the assumption of the continuous fluid, which is good for $R \gtrsim 100 l_{g}(=0.15 \mathrm{~m}$ at $0.5 \mathrm{AU}, 1.0 \mathrm{~m}$ at $1 \mathrm{AU}$, and $7 \mathrm{~m}$ for $2 \mathrm{AU})$. The values for $l_{g} \lesssim R \lesssim 100 l_{g}$ should be considered as rough approximations. Figure 4 shows that $R_{e}$ is on the order of 1 to 100 for a meter-sized spherical body. The values of $v_{\infty}$ given by Eq. (8) will be used to estimate the incident gas velocity relative to a SB as well as the collision velocity of a dust aggregate with a SB in the next section, and the value of $v_{r}$ given by Eq. (6) will be used to estimate the infall time scale of a SB in Section 4.

\section{Particle Trajectories Dragged by the Gas Flow}

As for gas flows around the sphere, the representative value of the Reynolds number is taken $R_{e}=50$, according to the above result. The following results are not sensitive to the value of $R_{e}$. The numerical code used in solving the gas flow field $v_{g}$ for $R_{e}=50$ is same as written in Takeda et al. (1985) and Takeda (1988), but we neglected the gravity of

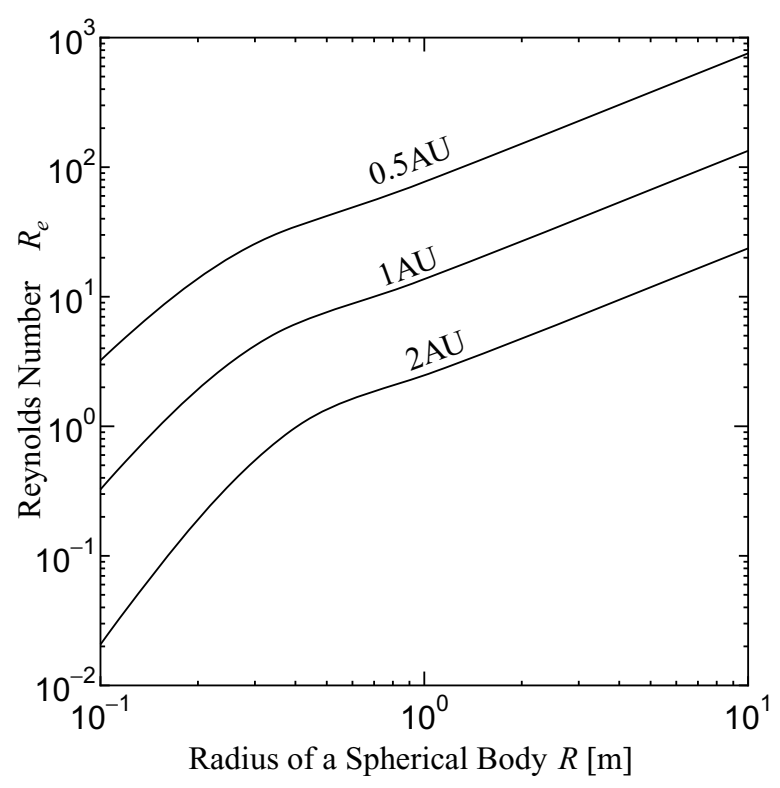

Fig. 4. The Reynolds number of the gas flow around a solid sphere with radius $R$, for $r=0.5,1$, and $2 \mathrm{AU}$.

the body. Although this is a code for a compressible flow, we solved the case where the Mach number is as small as 0.06 and the flow is almost incompressible. We take a coordinate system where the center of SB is at the origin. Gas flows in the $z$-direction from $z=-\infty$ with the velocity $v_{\infty}$ given by Eq. (8) above.

The equation of motion of a dust monomer or a dust aggregate is written

$$
d \boldsymbol{v}_{d} / d t=-\left(\boldsymbol{v}_{d}-\boldsymbol{v}_{g}\right) / t_{f}
$$

where $\boldsymbol{v}_{d}$ and $\boldsymbol{v}_{g}$ are dust and gas velocities, respectively, and $t_{f}$ is the friction time of the dust monomer or the dust aggregate. If the dust monomer is spherical with radius $r_{m o n}$ much smaller than $l_{g}$, the friction time is given by the Epstein Law:

$$
t_{f}=\rho_{\text {mat }} r_{\text {mon }} /\left(\rho_{g} c_{m o l} \delta p\right),
$$

where $\rho_{\text {mat }}$ is the material density of the dust particle, $c_{m o l}$ is the mean velocity of the gas molecules, and $\delta p$ is the momentum transfer coefficient ( $\delta p=1$ for specular reflection). For an aggregate of dust monomers (Blum et al., 1996; Blum and Wurm, 2000; Wurm et al., 2001a),

$$
t_{f}=3 m_{a g} /\left(4<\sigma_{a g}>\rho_{g} c_{m o l} \delta p\right)
$$

where $m_{a g}$ is the aggregate mass, $<\sigma_{a g}>$ is the geometric cross section (i.e., the mean projected area). In the following, we use non-dimensional variables normalizing the distance by $R$ and the velocity by $v_{\infty}$, and the time by $R / v_{\infty}$. The non-dimensional friction time is written by $T_{f}=t_{f} v_{\infty} / R$. The non-dimensional coordinates are written by large characters $(X, Y, Z)=(x / R, y / R, z / R)$. The non-dimensional distance from $Z$-axis is written by $\Pi=\left(X^{2}+Y^{2}\right)^{1 / 2}$.

The trajectories of a small dust aggregates are characterized by $T_{f}$ and $R_{e}$. The trajectories of small dust aggregates, whose initial positions are $Z_{0}=-10$ and $\Pi_{0}=0.1,0.2$, $0.3, \ldots, 1.0$, and the initial velocity is same as the gas velocity, are drawn in Figs. 5, 6 and 7 for $T_{f}=10,1.0$ and 0.1 , 


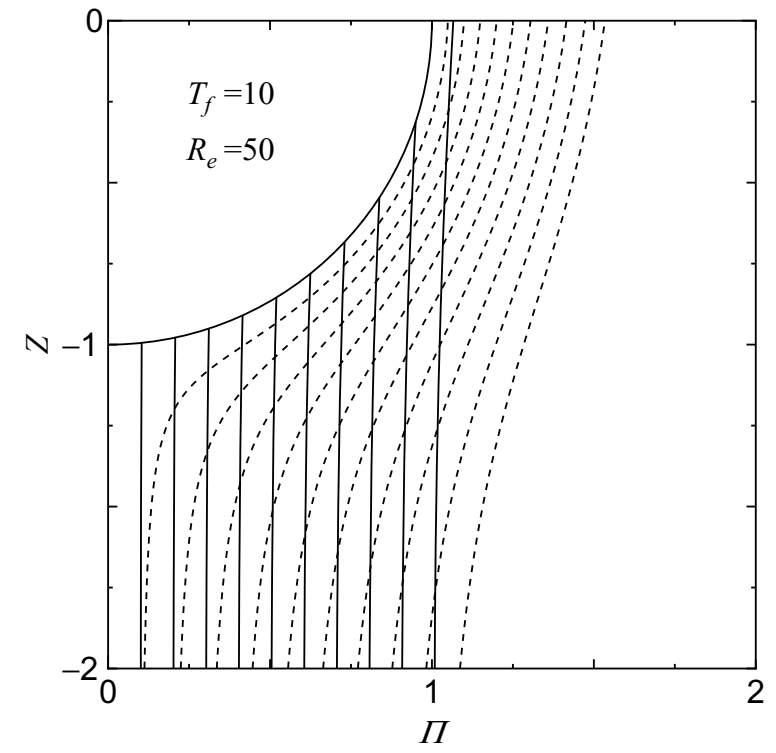

Fig. 5. Trajectories of small dust aggregates with non-dimensional friction time $T_{f}=10$ in the gas flow with $R_{e}=50$. The stream lines of gas are shown by dashed curves.

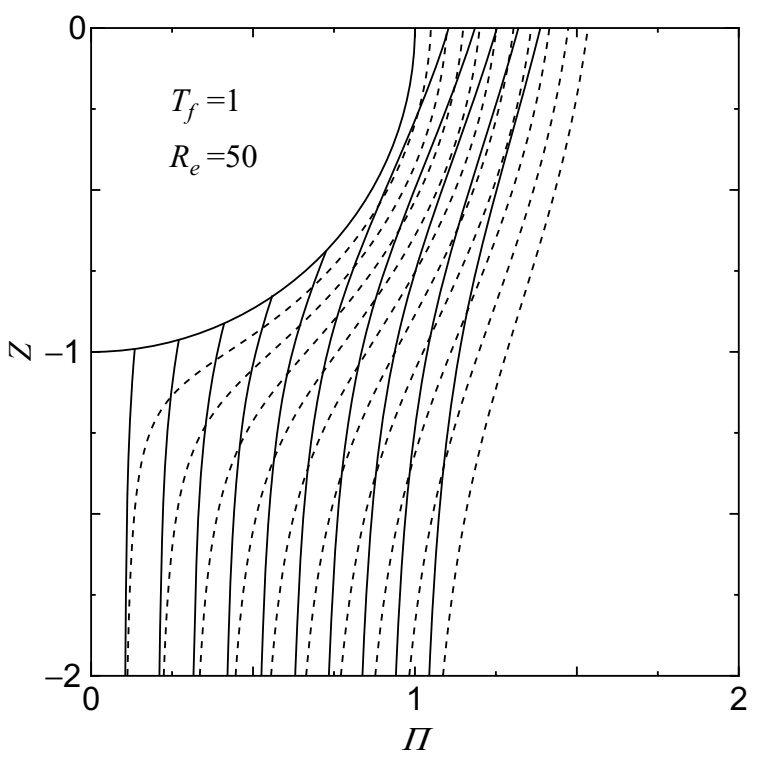

Fig. 6. Same as Fig. 5, but for $T_{f}=1$.

respectively. The Reynolds number is $R_{e}=50$. Numerical integration of Eq. (9) for a given $\boldsymbol{v}_{g}$ is made by using 8-th order Runge-Kutta method (Butcher, 1987). As seen from Fig. 5, the dust aggregates with $T_{f}=10$ are hardly affected by the gas flow. On the other hand, the dust aggregates with $T_{f}=0.1$ are nearly completely dragged by the gas flow and hardly hit the large sphere as seen from Fig. 7. For the dust aggregates with $T_{f}=1.0$, the trajectories are moderately affected by the gas drag and aggregates with impact parameter $\Pi_{0} \lesssim 0.5$ hit the sphere as seen from Fig. 6 . The maximum impact parameter for the collision is drawn as a function of $T_{f}$ in Fig. 8 for $R_{e}=50$. It is seen that dust aggregates can collide with a large sphere as long as $T_{f} \gtrsim 1$. This result is not sensitive to the value of $R_{e}$.

For a spherical monomer with the material density $\rho_{\text {mat }}$,

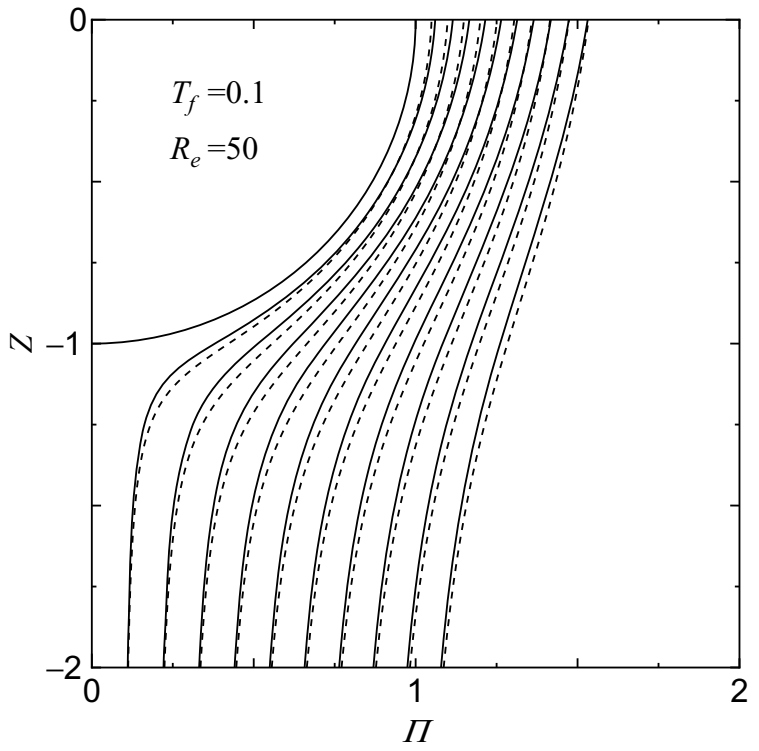

Fig. 7. Same as Fig. 5, but for $T_{f}=0.1$.

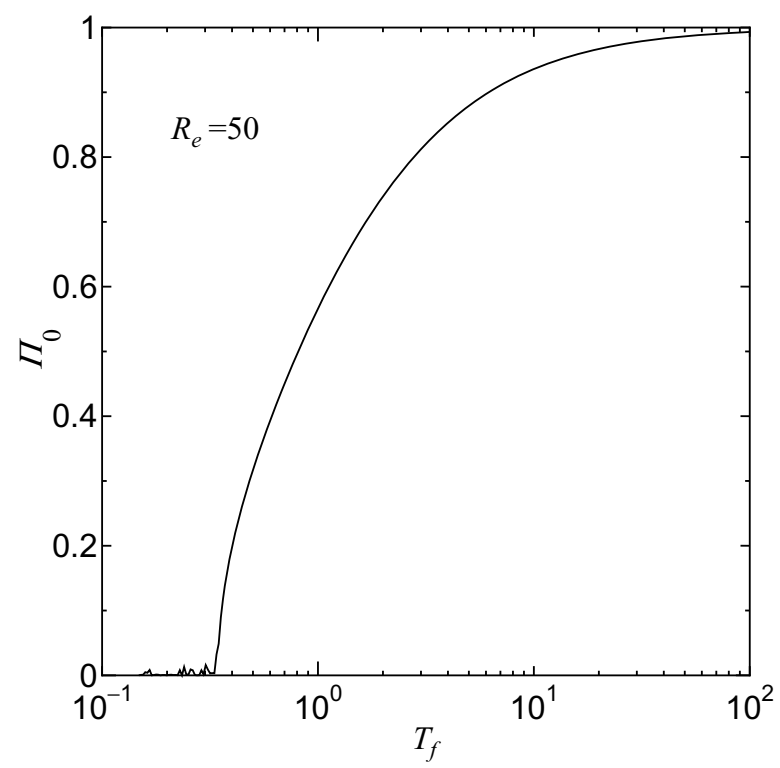

Fig. 8. The maximum value of the impact parameter $\Pi_{0}$ for collision with $\mathrm{SB}$ as a function of $T_{f}$.

the non-dimensional friction time is written as

$$
\begin{aligned}
T_{f}= & r_{\text {mon }} \rho_{\text {mat }} v_{\infty} /\left(\delta p \rho_{g} c_{m o l} R\right) \\
= & 50 \delta p^{-1}\left(\frac{r}{1 \mathrm{AU}}\right)^{3}\left(\frac{v_{\infty}}{50 \mathrm{~m} / \mathrm{s}}\right) \\
& \cdot\left(\frac{R}{1 \mathrm{~m}}\right)^{-1}\left(\frac{\rho_{\text {mat }}}{3 \times 10^{3} \mathrm{~kg} \mathrm{~m}^{-3}}\right)\left(\frac{r_{m o n}}{1 \mu \mathrm{m}}\right) .
\end{aligned}
$$

For a two-dimensional fluffy aggregate, $m_{a g} /<\sigma_{a g}>\sim$ $r_{\text {mon }} \rho_{\text {mat }}$ and the value of $T_{f}$ has the same order of magnitude as that of the monomer as seen from Eqs. (10) and (11). For a compact aggregate, the value of $T_{f}$ is larger. Thus inequality $T_{f} \gtrsim 1$ is always satisfied as long as $r \geq 1$ AU, $r_{m o n} \geq 1 \mu \mathrm{m}$ and $R \leq 10 \mathrm{~m}$. Although inequality $T_{f} \gtrsim 1$ is violated for the two-dimensional fluffy aggregate 


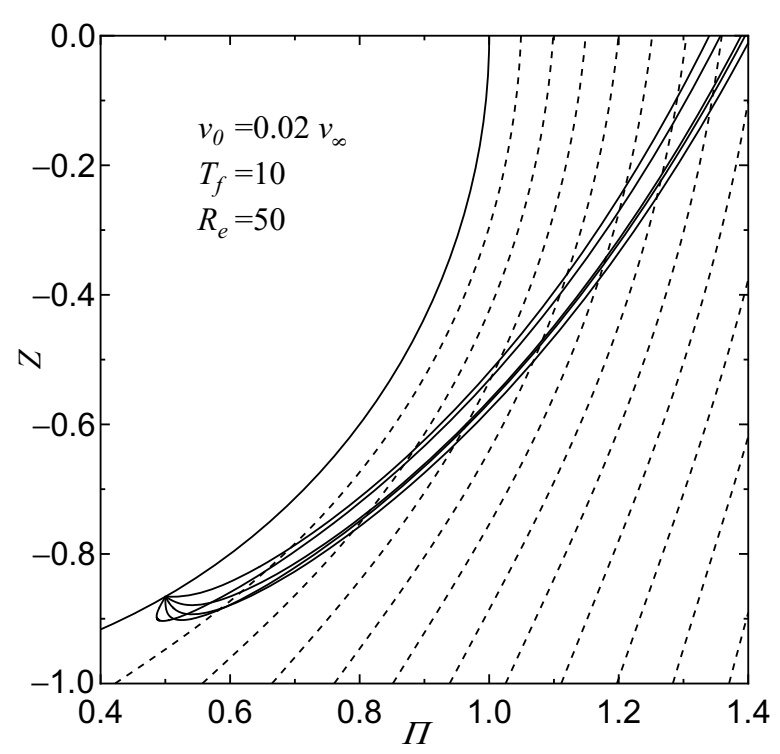

Fig. 9. Trajectories of monomers after break up on the surface of SB for $v_{0}=0.02 v_{\infty}, T_{f}=10$, and $R_{e}=50$. The stream lines of gas are shown by dashed curves.

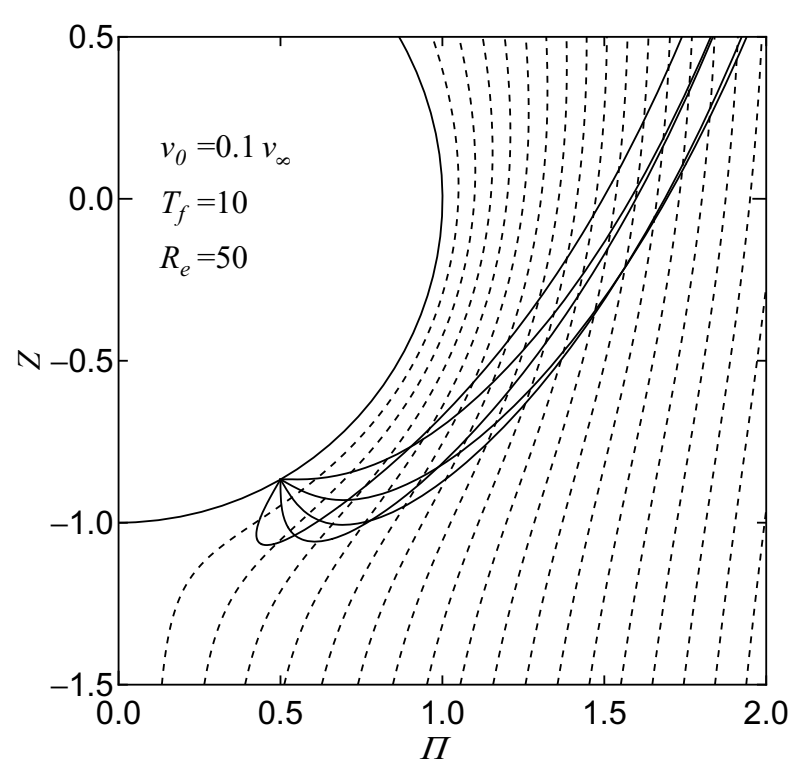

Fig. 10. Same as Fig. 9, but for $v_{0}=0.1 v_{\infty}$.

if $r<<1 \mathrm{AU}$, compaction of dust aggregate would increase $m_{a g} /<\sigma_{a g}>$ and the inequality would be satisfied. Thus, small dust aggregates would always hit large aggregates.

The collision velocity of dust aggregates with SB is on the order of $v_{\infty} \sim 50 \mathrm{~m} / \mathrm{s}$, as long as $T_{f} \gtrsim 1$. Then the small aggregate would be disrupted to monomers, which would bounce immediately (Dominik and Tielens, 1997; Blum and Wurm, 2000; Wurm et al., 2001a, b). We obtained the trajectories of these monomers assuming three values of the initial bounce velocity: $v_{0}=0.02 v_{\infty}, 0.1 v_{\infty}$ and $0.5 v_{\infty}$, i.e. $\sim 1$ $\mathrm{m} / \mathrm{s}, 5 \mathrm{~m} / \mathrm{s}$ and $25 \mathrm{~m} / \mathrm{s}$, respectively. The non-dimensional friction time is assumed $T_{f}=10$, i.e. $R=5 \mathrm{~m} / \mathrm{s}$ for $r_{\text {mon }}=1 \mu \mathrm{m}$ and $\rho_{\text {mat }}=3 \times 10^{3} \mathrm{~kg} \mathrm{~m}^{-3}$ at $r=1 \mathrm{AU}$. The initial position is set to be at $\Pi=0.5$. The initial angles between the velocity vectors and the normal of the surface are

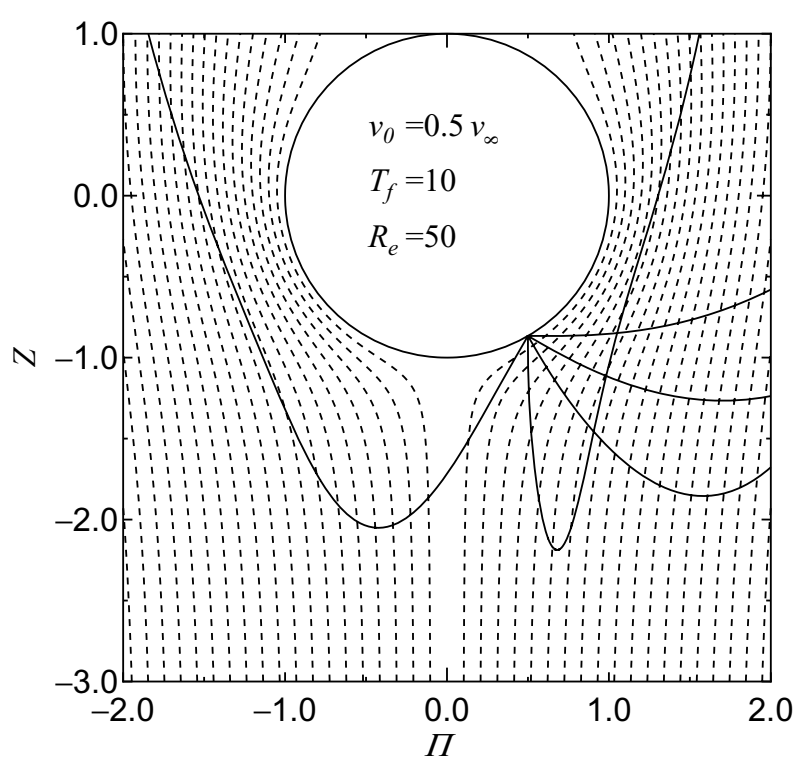

Fig. 11. Same as Fig. 9, but for $v_{0}=0.5 v_{\infty}$.

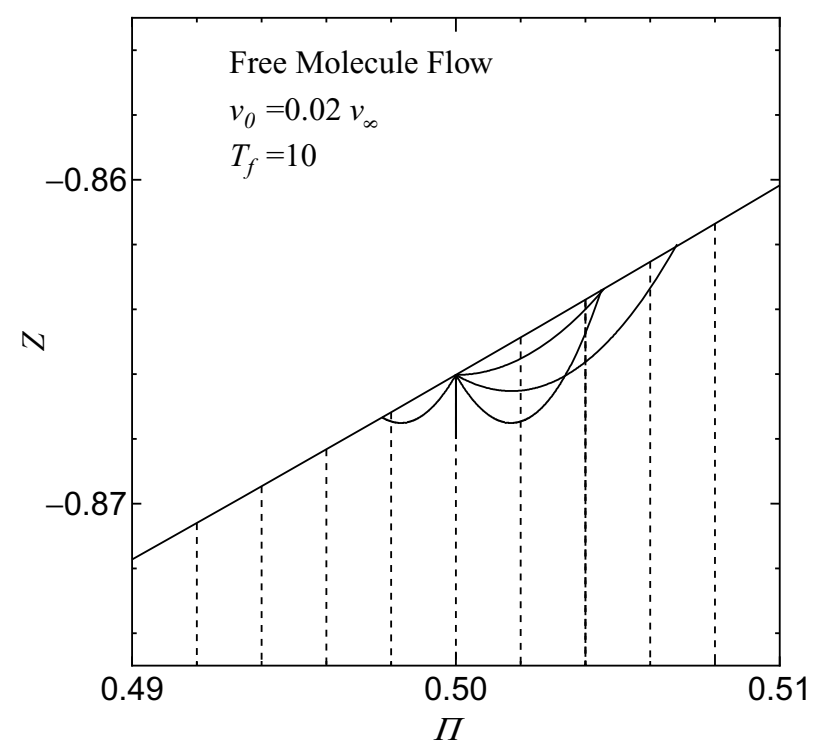

Fig. 12. Trajectories of monomers after break up on the surface of SB in free molecule flow with $v_{0}=0.02 v_{\infty}$ and $T_{f}=10$. The stream lines of gas are shown by dashed lines.

taken $0^{\circ}, \pm 30^{\circ}, \pm 60^{\circ}$. Figures 9 to 11 show the results for $v_{0}=0.02 v_{\infty}, 0.1 v_{\infty}$ and $0.5 v_{\infty}$, respectively. It is seen that all the monomers are swept away by the gas flow. We have made additional calculations for various values of parameters $v_{0}, T_{f}$, initial positions on $\mathrm{SB}$, and initial angles of the velocity vector. In all the cases, monomers are well dragged by the gas which flows away from SB, and no monomers hit the surface of SB again.

For comparison, the trajectories in free molecule flow are drawn in Figs. 12 to 14 , for $v_{0}=0.02 v_{\infty}, 0.1 v_{\infty}$ and $0.5 v_{\infty}$, respectively. It is seen that the monomers, which are launched from the surface of SB after a break up on the surface, return the surface again as long as $v_{0} \lesssim 0.1 v_{\infty}$. 


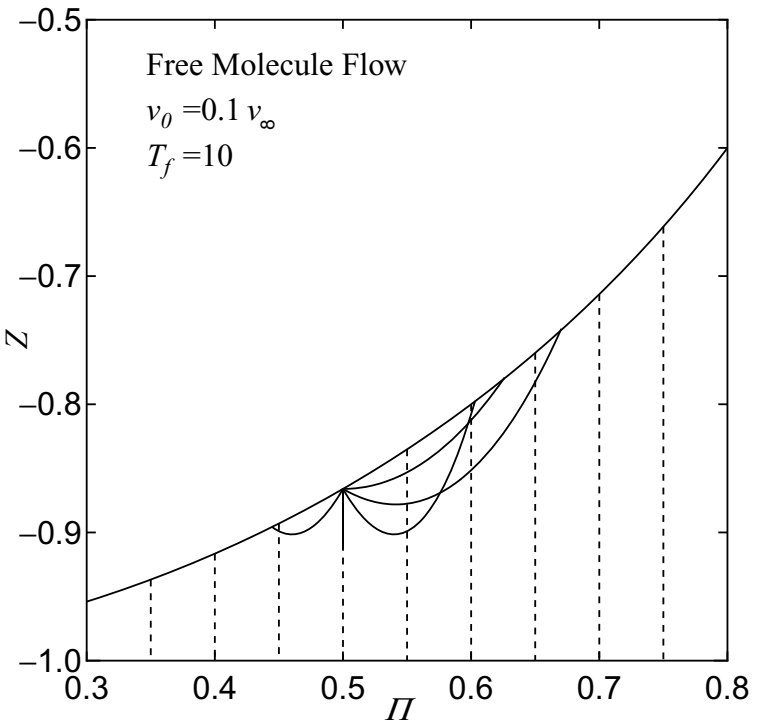

Fig. 13. Same as Fig. 12, but for $v_{0}=0.1 v_{\infty}$

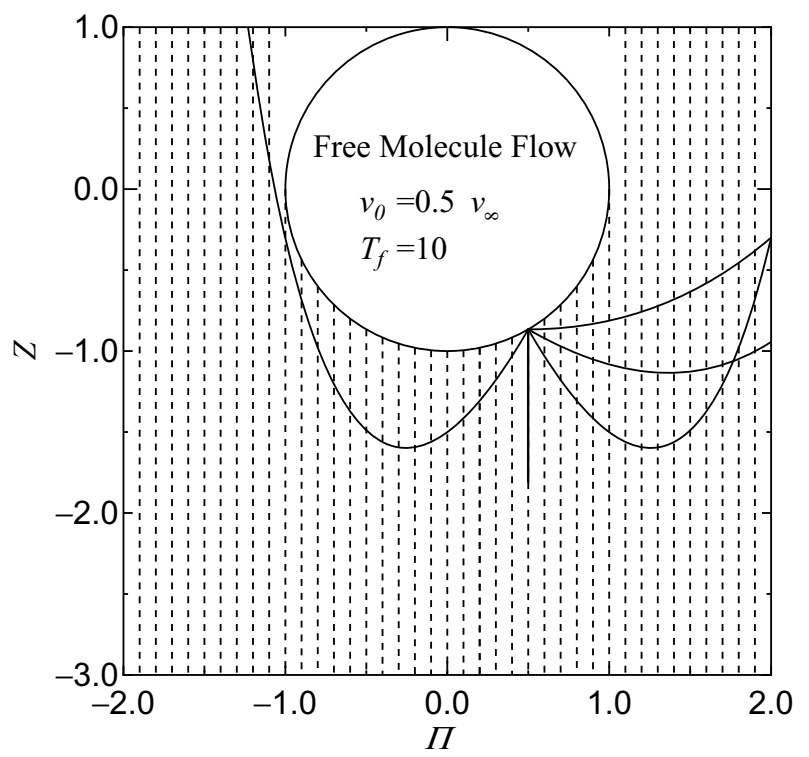

Fig. 14. Same as Fig. 12, but for $v_{0}=0.5 v_{\infty}$

\section{Discussion}

Here we discuss the growth of SBs in the solar nebula. If there are meter-sized SBs, they have velocities $\sim 50 \mathrm{~m} / \mathrm{s}$ relative to the nebular gas and small dust aggregates. Small dust aggregates hit the surface of SB with velocities $\sim 50$ $\mathrm{m} / \mathrm{s}$, and break up into dust monomers (Dominik and Tielens, 1997; Blum and Wurm, 2000). If $R \lesssim l_{g}$, some monomers hit the surface of SB again as shown in Figs. 12 and 13. If the velocity of the second collision is $\lesssim 1 \mathrm{~m} / \mathrm{s}$, the monomer sticks to SB (Heim et al., 1999; Poppe et al., 2000; Wurm et al., 2001a, b). Else if the collision velocity is $\gtrsim 1 \mathrm{~m} / \mathrm{s}$, the monomer bounces on the surface of SB, and loses some amount of the kinetic energy due to inelasticity. After several bouncing and losing energies, the monomer eventually sticks to $\mathrm{SB}$. Thus $\mathrm{SB}$ whose radius $R \lesssim 3 l_{g}$ grows by accreting monomers after collisional break up of dust aggregates (Wurm et al., 2001a, b). On the other hand, if $R \gtrsim 100 l_{g}$, the flow around SB is described by the continuous hydrodynamic flow. In this case, trajectories of monomers are as shown in Figs. 9 to 11, and they never hit the surface of SB again. Thus, such SB would not grow by accreting dust aggregates and monomers. It is then expected that the maximum radius of bodies made by dust accretion due to nongravitational forces is on the order of $10 l_{g}$ (see Fig. 1 as for the value of $l_{g}$ of the minimum mass solar nebula). In order to determine the maximum size of SB precisely, the detailed analysis based on the Boltzmann equation would be needed, which is beyond the scope of this paper.

It is interesting to note that the maximum size of SB in the asteroid region would be $\sim 1 \mathrm{~m}$ (see Fig. 1) for which the velocity towards the sun $\left|v_{r}\right|$ has the maximum value as large as several tens $\mathrm{m} / \mathrm{s}$ (see Fig. 3). The time scale of infall from $2 \mathrm{AU}$ to $1 \mathrm{AU}$ is about 100 yrs for SB with $0.4 \mathrm{~m}$. Thus it is possible that the shortage of matter in the asteroid region is due to effective infall, although it is usually believed that the giant planets' gravity cause the removal of planetesimals from the region (Wetherill, 1992; Chambers and Wetherill, 2001).

In this paper, we have shown that the formation of $\mathrm{km}$ sized planetesimals through the accretion of dust aggregates (Wurm et al., 2001a) is difficult to occur in the inner solar nebula ( $r \lesssim 5 \mathrm{AU}$ ) where the mean free path is less than $1 \mathrm{~m}$ (see Fig. 1). We cannot exclude the possibility of the planetesimal formation through dust accretion in the outer solar nebula ( $r \gtrsim 5 \mathrm{AU}$ ) by our simulations which assumes the continuous flows around SBs. Particularly, in the outermost part of the solar nebula where comets were formed ( $r \gtrsim 30 \mathrm{AU})$, planetesimals were probably formed through collisional coagulations (Weidenschilling, 1997). Elaborate numerical simulations of the flow around SBs using the Boltzmann equation as well as experiments in the transition regime between the free molecular flow and the continuous flow should be done in future in order to elucidate the dust accretion processes by SBs in the outer solar nebula.

Now, we consider how planetesimals were formed in the inner solar nebula. Sekiya (1998) have shown that the increase of the dust/gas surface density ratio suppresses the shear-induced turbulence. Thus, the gravitational instability might occur if there is a mechanism to concentrate dust to some locations (Youdin and Shu, 2002). One possibility is the above mentioned effective infall of meter-sized body from asteroid region, which supplies some additional mass to the region around $1 \mathrm{AU}$. Another possibility is the concentration of dust aggregates by eddies which might be persistent in the solar nebula like Jovian great red spot (Barge and Sommeria, 1995; Tanga et al., 1996; Chavanis, 2000; Godon and Livio, 2000; Marcos and Barge, 2001; Klahr and Bodenheimer, 2003). Further, the UV irradiation (Shu et al., 1993 ) and the magneto-rotational instability around the disk surface region (Gammie, 1996) lead the loss of the gas from the nebula leaving the dust around the midplane, which increases the dust/gas surface density ratio. Recently, Ishitsu and Sekiya (2003) have shown that the tidal force has the effect to suppress the shear instability, which also act for the gravitational instability. Anyway, more works should be done in future to elucidate the formation mechanism of planetesimals. 


\section{Conclusion}

In this paper, the growth of meter-sized bodies in the solar nebula by dust accretion is examined. Meter-sized bodies have velocity about $50 \mathrm{~m} / \mathrm{s}$ relative to the gas. Small dust aggregates in the nebula have almost same velocity with the gas. Thus, the incident velocity of a small dust aggregate toward a meter-sized body is about $50 \mathrm{~m} / \mathrm{s}$. When the small dust aggregate hits a meter-sized body, the aggregate breaks into $\mu \mathrm{m}$-sized dust monomers. These monomers accrete onto the body after several bouncing as proposed by Wurm et al. (2001a), if the mean free path of the gas molecules is larger than the radius of the body. On the other hand, hydrodynamic flow around the body prevents monomers from hitting the surface of the body again and the body will not grow further, if the body is much larger than the mean free path of the molecules. Thus, the sizes of bodies are limited to the order of 10 times the mean free path. Kilometer-sized planetesimals were hardly formed by dust accretion within 5 $\mathrm{AU}$ from the sun where the mean free path of gas molecules are smaller than $1 \mathrm{~m}$; they were probably formed by the gravitational instabilities.

Acknowledgments. The authors thank G. Wurm and Y. Nakagawa for several constructive comments, and the Astronomical Data Analysis Center of the National Astronomical Observatory of Japan and Academic Center for Computing and Media Studies of Kyoto University for the use of computers.

\section{References}

Adachi, I., C. Hayashi, and K. Nakazawa, The gas drag effect on the elliptic motion of a solid body in the primordial solar nebula, Progr. Theor. Phys., 56, 1756-1771, 1976.

Barge, P. and J. Sommeria, Did planet formation begin inside persistent gaseous vortices?, Astron. Astrophys., 295, L1-L4, 1995.

Blum, J. and G. Wurm, Experiments on sticking, restructuring, and fragmentation of preplanetary dust aggregates, Icarus, 143, 138-146, 2000.

Blum, J., G. Wurm, S. Kempf, and T. Henning, The Brownian motion of dust particles in the solar nebula: An experimental approach to the problem of pre-planetary dust aggregation, Icarus, 124, 441-451, 1996.

Butcher, J. C. The Numerical Analysis of Ordinary Differential Equations. Runge-Kutta and General Linear Methods, 512 pp., Wiley, New York, 1987.

Chambers, J. E. and G. W. Wetherill, Planets in the asteroid belt, Meteorit Planet. Sci., 36, 381-399, 2001.

Chavanis, P. H., Trapping of dust by coherent vortices in the solar nebula, Astron. Astrophys., 356, 1089-1111, 2000.

Coradini, A., C. Federico, and G. Magni, Formation of planetesimals in an evolving protoplanetary disk, Astron. Astrophys, 98, 173-185, 1981.

Cuzzi, J. N., A. R. Dobrovolskis, and J. M. Champney, Particle-gas dynamics in the midplane of a protoplanetary nebula, Icarus, 106, 102-134, 1993.

Dominik, C. and A. G. G. M. Tielens, The physics of dust coagulation and the structure of dust aggregates in space, Astrophys. J., 480, 647-673, 1997.

Gammie, C. F., Layered accretion in T Tauri disks, Astrophys. J., 457, 355 362,1996

Godon, P. and M. Livio, The formation and role of vortices in protoplanetary disks, Astrophys. J., 537, 396-404, 2000.

Goldburg, A. and B. H. Florsheim, Transition and Strouhal number for the incompressible wake of various bodies, Phys. Fluids, 9, 45-50, 1966.

Goldreich, P. and W. R. Ward, The formation of planetesimals, Astrophys. J., 183, 1051-1061, 1973.

Hayashi, C., Structure of the solar nebula, growth and decay of magnetic fields, and effects of magnetic and turbulent viscosities on the nebula, Progr. Theor. Phys. Suppl., 70, 35-53, 1981.

Hayashi, C., K. Nakazawa, and Y. Nakagawa, Formation of the solar system, in Protostars and Planets II, edited by D. C. Black and M. S. Matthews, pp. 1100-1153, Univ. of Arizona Press, Tucson, 1985.

Heim, L.-O., J. Blum, M. Preuss, and H.-J. Butt, Adhesion and friction forces between spherical micrometer-sized particles, Phys. Rev. Lett., 83, 3328-3331, 1999

Ishitsu, N. and M. Sekiya, The effects of the tidal force on the shear instabilities in the dust layer of the solar nebula, Icarus, 2003 (in press).

Kalro, V. and T. Tezduyar, 3D computation of unsteady flow past a sphere with a parallel finite element method, Comput. Methods in Appl. Mech. Engrg., 151, 267-276, 1998.

Klahr, H. H. and P. Bodenheimer, Turbulence in accretion disks: Vorticity generation and angular momentum transport via the global baroclinic instability, Astrophys. J., 582, 869-892, 2003.

Lee, S., A numerical study of the unsteady wake behind a sphere in a uniform flow at moderate Reynolds numbers, Comp. Fluids, 29, 639$667,2000$.

Magarvey, R. H. and R. L. Bishop, Transition ranges for three-dimensional wakes, Can. J. Phys., 39, 1418-1422, 1961.

Marcos, C. D. and P. Barge, The effect of long-lived vortical circulation on the dynamics of dust particles in the mid-plane of a protoplanetary disc, Mon. Not. R. Astron. Soc., 323, 601-614, 2001.

Nakagawa, Y., M. Sekiya, and C. Hayashi, Settling and growth of dust particles in a laminar phase of a low-mass solar nebula, Icarus, 67, 375390, 1986.

Nakamura, I., Steady wake behind a sphere, Phys. Fluids, 19, 5-8, 1976.

Poppe, T., J. Blum, and T. Henning, Analogous experiments on the stickiness of micron-sized preplanetary dust, Astrophys. J., 533, 454-471, 2000 .

Rimon, Y. and S. I. Cheng, Numerical solution of a uniform flow over a sphere at intermediate Reynolds numbers, Phys. Fluids, 12, 949-959, 1969.

Safronov, V. S., Evolution of the Protoplanetary Cloud and Formation of the Earth and the Planets, Nauka, Moscow, [NASA Tech. Trans. F-677], 1969.

Sekiya, M., Gravitational instabilities in a dust-gas layer and formation of planetesimals in the solar nebula, Progr. Theor. Phys., 69, 1116-1130, 1983.

Sekiya, M., Quasi-equilibrium density distributions of small dust aggregations in the solar nebula, Icarus, 133, 298-309, 1998.

Shu, F. H., D. Johnstone, and D. Hollenbach, Photoevaporation of the solar nebula and the formation of the giant planets, Icarus, 106, 92-101, 1993.

Takeda, H., Drag on a gravitating body, Progr. Theor. Phys. Suppl., 96, 196210, 1988

Takeda, H., T. Matsuda, K. Sawada, and C. Hayashi, Drag on a gravitating sphere moving through a gas, Progr. Theor. Phys., 74, 272-287, 1985.

Taneda, S., Experimental investigation of the wake behind a sphere at low Reynolds numbers, J. Phys. Soc. Japan, 11, 1104-1108, 1956.

Tanga, P., A. Babiano, B. Dubrulle, and A. Provenzale, Forming planetesimals in vortices, Icarus, 121, 158-170, 1996.

Weidenschilling, S. J., Aerodynamics of solid bodies in the solar nebula, Mon. Not. Roy. Astron. Soc., 180, 57-70, 1977.

Weidenschilling, S. J., Dust to planetesimals: Settling and coagulation in the solar nebula, Icarus, 44, 172-189, 1980.

Weidenschilling, S. J., Evolution of grains in a turbulent solar nebula, Icarus, 60, 553-567, 1984.

Weidenschilling, S. J., The origin of comets in the solar nebula: A unified model, Icarus, 127, 290-306, 1997.

Weidenschilling, S. J. and J. N. Cuzzi, Formation of planetesimals in the solar nebula, in Protostars and Planets III, edited by E. H. Levy and J. I. Lunine, pp. 1031-1060, Univ. of Arizona Press, Tucson, 1993.

Wetherill, G. W., An alternative model for the formation of the asteroids, Icarus, 100, 307-325, 1992.

Wurm, G., J. Blum, and J. E. Colwell, A new mechanism relevant to the formation of planetesimals in the solar nebula, Icarus, 151, 318-321, 2001a.

Wurm, G., J. Blum, and J. E. Colwell, Aerodynamical sticking of dust aggregates, Phys. Rev., E64, 46301-46309, 2001b.

Youdin, A. N. and F. H. Shu, Planetesimal formation by gravitational instability, Astrophys. J., 580, 494-505, 2002.

M. Sekiya (e-mail: sekiya@geo.kyushu-u.ac.jp) and H. Takeda 